\title{
Simulation-based equation of state of the hard disk fluid and prediction of higher-order virial coefficients
}

\author{
J. KOLAFA* and M. ROTTNER \\ Department of Physical Chemistry, Prague Institute of Chemical Technology, 16628 Praha 6, Czech Republic \\ (Received 00 Month 200x; In final form 00 Month 200x)
}

\begin{abstract}
We present new molecular dynamics results for the pressure of the pure hard disk fluid up to the hexatic transition (about reduced density 0.9 ). The data combined with the known virial coefficients (up to $B_{10}$ ) are used to build an equation of state, to estimate higher-order virial coefficients, and also to obtain a better value of $B_{10}$. Finite size effects are discussed in detail. The "van der Waals-like" loop reported in literature in the vicinity of the fluid/hexatic transition is explained by suppressed density fluctuations in the canonical ensemble. The inflection point on the pressure-density dependence is predicted by the equation of state even if the hexatic phase simulation data are not considered.
\end{abstract}

Keywords: hard disk, equation of state, virial coefficients, fluid phase, hexatic phase, finite-size effects

\section{Introduction}

The hard disk fluid is the simplest model of various surface systems (surfactants, adsorption of molecules on smooth surfaces). It has been intensively studied by a number of methods, of which we are interested in Monte Carlo and molecular dynamics simulations [1, 2, 3, 4, virial coefficients [5, 6, and equations of state (EOS) [7, 8, 9, 10, 11, 12, 13, 14, 15, 16, 17, 18. Recently, peculiarities of phase transitions from fluid to hexatic to solid are frequently studied [18,4].

In this work we focus mainly on the fluid region. The fluid phase changes at density of about $\rho_{\mathrm{c}}=$ 0.8995(23) ( $\rho$ denotes the reduced number density, $\rho=N \sigma^{2} / A$, where $N$ is the number of disks, $\sigma$ their diameter, and $A$ the system area) to the hexatic phase [19,20, 18 .

\section{Methods}

\section{$2.1 \quad$ Simulation methodology}

2.1.1 Molecular dynamics code. To obtain accurate data on the EOS, we use standard molecular dynamics (MD) simulations in the microcanonical ensemble and tetragonal (square) periodic boundary conditions with zero total momentum (MD-NVE; we keep the usual abbreviation NVE for constant Number of particles-Volume-Energy even if the volume is here replaced by the area). Our MD program combines the ideas of the linked-cell list method both in space and time [1,2 and is highly optimized. Details on the code are given in our previous paper [21]. In the present study, we use $N=4000$ and 9000, and at higher densities 16000,25000 and 50000 disks of unit diameter in a square periodic box.

2.1.2 Compressibility factor. The compressibility factor $Z=p A /(N k T)=p /(\rho k T)(p$ denotes pressure, $k$ the Boltzmann constant, and $T$ absolute temperature) can be calculated from MD simulations in

\footnotetext{
*Corresponding author. Email: jiri.kolafa@vscht.cz
} 


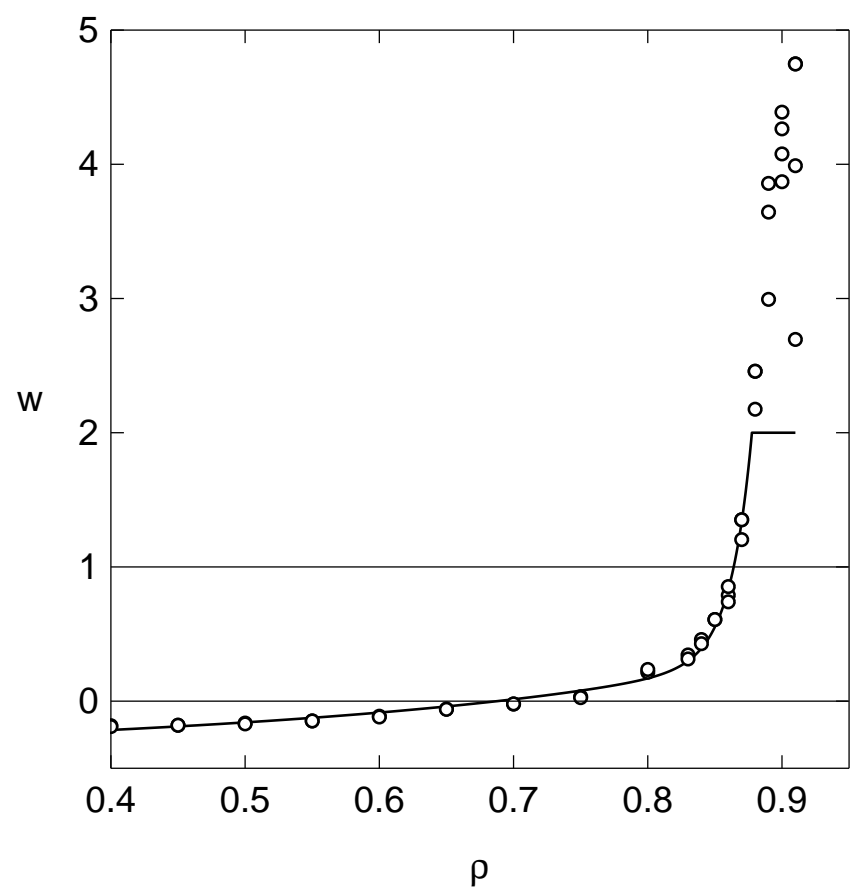

Figure 1. Weights $w$ for mixing both formulas for $Z$ in equation (4) and the fit bounded by $w \leq 2$.

two ways [1,2]. One formula uses the virial of force

$$
Z_{\mathrm{vir}}\left(t_{1}, t_{2}\right)=1-\frac{N-1}{N} \frac{1}{2 E_{\mathrm{kin}}\left(t_{2}-t_{1}\right)} \sum_{t \in\left(t_{1}, t_{2}\right)} \Delta \mathbf{v}_{i j} \cdot \mathbf{r}_{i j}
$$

where the sum is over all collisions occurring during time interval $\left(t_{1}, t_{2}\right), \Delta \mathbf{v}_{i j}$ is the change of velocity of both colliding disks of mutual position $\mathbf{r}_{i j}$, and $E_{\text {kin }}$ is the kinetic energy (constant in the MD-NVE simulation). The alternative formula uses the collision rate

$$
Z_{\text {rate }}\left(t_{1}, t_{2}\right)=1+\gamma(N) \sqrt{\frac{\pi}{2 D N E_{\text {kin }}}} \frac{1}{t_{2}-t_{1}} \sum_{t \in\left(t_{1}, t_{2}\right)} 1,
$$

where $\sum_{t \in\left(t_{1}, t_{2}\right)} 1$ is the number of collisions in time interval $\left(t_{1}, t_{2}\right)$ and $[2$

$$
\gamma(N)=\frac{\Gamma[(D(N-1)+1) / 2]}{\Gamma[D(N-1) / 2](D N / 2)^{1 / 2}}
$$

where $D=2$ is the dimensionality.

Both formulas give within statistical inaccuracies the same values, but their statistical errors differ. Our final result is therefore a weighed average of both formulas,

$$
Z=w Z_{\mathrm{vir}}+(1-w) Z_{\text {rate }}
$$

where the mixing weight $w=w(\rho)$ is a function of density. Methodology for determining the optimum function $w$ has been explained in detail in [21. The simulation results on $w(\rho)$ are shown in figure 1 along with a function fitted to the data and bounded by $w \leq 2$ to avoid too extrapolated values. This function was used in final analysis of MD data to avoid bias. 


\begin{tabular}{|c|c|c|}
\hline$\rho$ & $Z$ & $\sigma(Z)$ \\
\hline 0.40 & 2.1514393 & 0.0000052 \\
\hline 0.45 & 2.4276680 & 0.0000065 \\
\hline 0.50 & 2.7601235 & 0.0000079 \\
\hline 0.55 & 3.1647878 & 0.0000098 \\
\hline 0.60 & 3.663691 & 0.000012 \\
\hline 0.65 & 4.287926 & 0.000015 \\
\hline 0.70 & 5.082362 & 0.000019 \\
\hline 0.75 & 6.113391 & 0.000026 \\
\hline 0.80 & 7.476491 & 0.000036 \\
\hline 0.83 & 8.494891 & 0.000050 \\
\hline 0.84 & 8.866011 & 0.000059 \\
\hline 0.85 & 9.245785 & 0.000072 \\
\hline 0.86 & 9.621609 & 0.000082 \\
\hline 0.87 & 9.96782 & 0.00013 \\
\hline $0.88^{\mathrm{a}}$ & 10.2309 & 0.0003 \\
\hline 0.89 & 10.3176 & 0.0011 \\
\hline $0.90^{\mathrm{b}}$ & 10.2059 & 0.0011 \\
\hline \multicolumn{3}{|c|}{ compromise between } \\
\hline \multicolumn{3}{|c|}{$\begin{array}{l}10.23095(26) \text { by } \quad(5) \text { and } \\
\begin{array}{l}10.23055(54) \\
\text { trapolation. }\end{array} \text { by } Z(1 / N) \text { ex- }\end{array}$} \\
\hline
\end{tabular}

2.1.3 Start and equilibration. The initial configuration was a tetragonal crystal with random velocities assigned to particles. This setup creates a disorder within a few collisions. At higher densities, a locally hexagonal arrangement gradually develops. Therefore a period of equilibration follows until the compressibility factor reaches a constant value with fluctuations. At higher densities and for a large system this takes a long time.

\section{$2.2 \quad$ Finite size effects}

2.2.1 Fluid region. The finite-size errors are in our pseudoexperimental setup at low densities several times larger than the statistical errors. E.g., at the lowest density simulated, $\rho=0.4$, one would need as many as $N=160000$ particles to guarantee the same systematic finite-size error as the statistical error of table 1 It is more efficient to simulate a smaller system and to correct for the finite-size error to obtain the thermodynamic limit $(N \rightarrow \infty)$ because correlation times and therefore convergence in large two-dimensional systems is slow. At about $\rho=0.85$ both errors become comparable for $N=20000$. In a vicinity of the hexatic transition the finite-size errors become large again and difficult to determine.

The correction procedure can be divided into three steps [21,22]:

$$
\mathrm{MD}-\mathrm{NVE} \longrightarrow \mathrm{NVT} \longrightarrow \mu \mathrm{VT} \longrightarrow \text { thermodynamic limit }
$$

where the first step is already included in formulas (11) and (21), and $\mu \mathrm{VT}$ stands for the grand-canonical ensemble.

The largest source of inaccuracy for hard-body systems is the second step, NVT $\rightarrow \mu \mathrm{VT}$ [23, 24]. This "ensemble correction" is caused by suppressed fluctuations of density (number of particles) in the canonical ensemble and is given by 21,25$]$

$$
Z_{\mu \mathrm{VT}}-Z_{\mathrm{NVT}}=\frac{\rho}{2 N} \frac{\partial^{2} p}{\partial \rho^{2}}\left[\frac{\partial p}{\partial \rho}\right]^{-1}+\mathcal{O}\left(N^{-2}\right)
$$

where indices $\mu \mathrm{VT}$ and NVT refer to the respective expectation values in the grand-canonical and canonical 
ensembles at the same number density (i.e., $\langle N\rangle_{\mu \mathrm{VT}}=N_{\mathrm{NVT}}$ ). This term requires knowledge of an EOS which is not known in advance. The correction procedure is therefore self-consistent (we start with no correction, fit the EOS, calculate the correction and better data, etc.), albeit rapidly converging. For $\rho>0.88$, the accuracy of the EOS is not sufficient to calculate the second derivative needed in (5) and we use linear extrapolation of $Z$ in dependence on $1 / N$ to zero. In a close vicinity of $\rho_{\mathrm{c}}$ even this approach fails.

2.2.2 Near the critical point. It was investigated in detail in [18 that the $p(\rho)$ dependence has an inflection point at the fluid/hexatic transition, $p=Z k T \rho=p_{0}-$ const $\times\left(\rho_{\mathrm{c}}-\rho\right)^{\alpha^{\prime}}$ as $\rho \rightarrow \rho_{\mathrm{c}}$, where $\alpha^{\prime}=4.55$. Then the "ensemble error" (of opposite sign than the ensemble correction) becomes

$$
Z_{\mathrm{NVT}}-Z_{\mu \mathrm{VT}}=\frac{\alpha^{\prime}-1}{\rho_{\mathrm{c}}-\rho} \frac{\rho}{2 N}
$$

and similarly for $\rho>\rho_{\mathrm{c}}$ (likely with a different critical exponent $\alpha^{\prime}$ ). This term is positive for $\rho<\rho_{\mathrm{c}}$, negative for $\rho>\rho_{\mathrm{c}}$, and it diverges at $\rho_{\mathrm{c}}$. For $\rho=0.895$ and $N=256^{2}$ we obtain from (6) the NVT pressure by 0.005 higher than the grand-canonical one which is in qualitative agreement with the value of 0.01 by [4] (the statistical error is not provided, but it is likely less than 0.01 ). The correct value of the correction may be affected by higher order terms; apparently this must be true in a close vicinity of $\rho_{\mathrm{c}}$ because the ensemble correction cannot diverge at finite $N$. Therefore the "van der Waals-like" loop [3] is an artifact caused by suppressed density fluctuations in the constant- $N$ ensemble and it disappears if data are free of finite-size effects.

Near the first-order phase transition (occuring e.g. for hard spheres) one observes a hysteresis caused by long-lived metastable states rather than a "loop"; a loop can arise at very small systems and for long runs so that an equilibrium is maintained. There is no true hysteresis near the continuous transition point, although of course the dynamics (and convergence) slows down.

\section{$2.3 \quad$ Equation of state}

2.3.1 Correlation of the data. The pseudoexperimental data were fitted to a polynomial in $x=y /(1-$ $y$ ), where $y=A_{\mathrm{HD}} \rho$ is the packing fraction and $A_{\mathrm{HD}}$ is the disk area. The compressibility factor reads as

$$
Z(y)=\sum_{i=0}^{k} A_{i}\left(\frac{y}{1-y}\right)^{i}
$$

where $A_{0}-A_{4}$ are determined so that virial coefficients $B_{2}-B_{5}$, known either analytically [26]27] or $\left(B_{5}\right.$ [28] with high precision, are exactly reproduced. Parameters $A_{i}, 4<i \leq k$, are adjustable, but some of them may be zero. The number of degrees of freedom (number of all data minus number of adjustable parameters) is denoted $n_{\text {free }}$.

This choice needs some explanation. Terms $y /(1-y)$ appear in several theories for both hard spheres and hard disks: the scaled particle theory [29], Percus-Yevick and hypernetted-chain integral equations [30], and resummation of approximated virial coefficients (Carnahan-Starling equation) 31]. The main reason for this form is that it moderates the sharp increase of $Z$ with increasing $y$ as suggested in [32] (equation (13)) under name $y$-expansion (function $y$ is here called $x$ and should not be confused with packing fraction $y$ ).

Another popular choice is a rational function which is closely related to the Padé approximant 33. derived from a formal virial power series; the coefficients are determined by the least-square method. There is some experience with this method in our laboratory 34, but detailed investigation including high densities found this method numerically unstable and less flexible; we have not checked this approach for hard disks, though. In addition, we have theoretical objections against interpretation of the pole (zero point of the denominator) of such a rational function as the random close packing of the hard sphere fluid 
(frozen glass). This interpretation assumes that the EOS can be analytically continued beyond the freezing point which is not true [35, 36, 37; ; it can be continued with a limited precision only - the random close packing is an inaccurate concept in principle. With increasing precision of input data this inaccuracy may cause problems in fitting. In the hard disk system it is impossible to extrapolate beyond the continuous transitions (with inflection points on the pressure-density dependence) to get any random close packing and even this inaccurate reason for using rational functions becomes invalid.

Another consequence of the nonanalyticity is that the radius of convergence of the virial series is less than or equal to the phase transition density (first-order freezing of the hard sphere fluid or continuous transition of the hard disk fluid). Consequently the approximately quadratic $B_{n}^{*}$ dependence (for hard spheres) and linear dependence (for hard disks), related to expanded $y /(1-y)$ terms, cannot extent to infinite $n$. It is in principle possible to determine the radius of convergence from the sequence of $B_{n}$, but the available precision and the number of terms are not sufficient.

With functional form (7), the standard objective function

$$
s^{2}=\frac{1}{n_{\text {free }}}\left\{\sum_{j=1}^{n_{\text {data }}}\left[\frac{Z\left(y_{j}\right)-Z_{j}}{\sigma\left(Z_{j}\right)}\right]^{2}+\sum_{j=6}^{10}\left[\frac{B_{j}^{\mathrm{EOS}}-B_{j}^{\mathrm{MC}}}{\sigma\left(B_{j}^{\mathrm{MC}}\right)}\right]^{2}\right\}
$$

was minimized, where $\sigma$ stands for the standard error, $B_{j}^{\mathrm{EOS}}$ is the virial coefficient calculated from the EOS and $B_{j}^{\mathrm{MC}} \pm \sigma\left(B_{j}^{\mathrm{MC}}\right)$ are virial $\mathrm{MC}$ data with standard errors [5, 6]. In other words, both the MD data on the compressibility factor and the MC data on the virial coefficient are correlated simultaneously.

The value of $s$ for an optimum fit is around unity provided that the input standard errors $\sigma$ are reliable, which is the case of our simulations where $\sigma$ is determined with accuracy (error of error) of a few per cent [21]. If $s \gg 1$ then the number of adjustable parameters is not sufficient do describe the data. On the other hand, one should not use more parameters than necessary because just noise would be fitted; the best test is to remove one parameter and to observe whether $s$ significantly increases.

2.3.2 Higher-order virial coefficients. Expanding equation (17) in powers of density gives virial coefficients. Virial coefficients $B_{i}$ for $i \leq 5$ are exactly reproduced, for $6 \leq i \leq 10$ they are modified because their change is allowed by a simultaneous fit (within statistical errors), and for $i>10$ they are predicted.

\section{Results}

\subsection{Molecular dynamics}

First, we have checked that both the virial (11) and rate (2) routes to the compressibility factor are equivalent. The differences are within combined statistical errors the same and only for two systems with $N=9000$ and $\rho=0.7$ and $\rho=0.75$ slightly exceed one standard deviation. In fact, because of correlations in the data, the difference is at low and large densities much less than the combined standard error.

In order to assess the role of the periodic errors, we fitted the total correlation function $h(r)=g(r)-1$ at large separations to attenuated oscillations, $h(r)=\operatorname{Re}[A \exp (-B r) / r]$, where $A$ and $B$ are complex constants and $\operatorname{Re}$ denotes the real part. The value of $\operatorname{Re}(B)$ describes the decay of correlations and its typical value at simulation square size $L=A^{1 / 2}, \operatorname{Re}(A) \exp [-\operatorname{Re}(B) L] / L$, is the estimate of the periodic error. We found that $\operatorname{Re}(B)=0.22$ for $\rho=0.88$. Consequently the periodic error is negligible for all used $N$ and $\rho \leq 0.88$, and therefore formula (5) or linear $Z(1 / N)$ dependence is sufficient to account for finite size effects. For $\rho=0.89$ we found $\operatorname{Re}(B)=0.11$ and $N=4000$ may have a small periodic error about $2 \cdot 10^{-5}$; the datum was nevertheless discarded. For $\rho=0.90 \approx \rho_{\mathrm{c}}$ it holds $\operatorname{Re}(B)=0.044$ and even $N=16000$ is barely sufficient.

For $\rho<0.88$, the data for different $N$ were corrected by (5) and a weighed average was taken; a check was made that the corrected data do match within statistical errors. Correction term (5) is not applicable for $\rho \geq 0.89$ because it contains the second derivative of the EOS and therefore the correction term is 


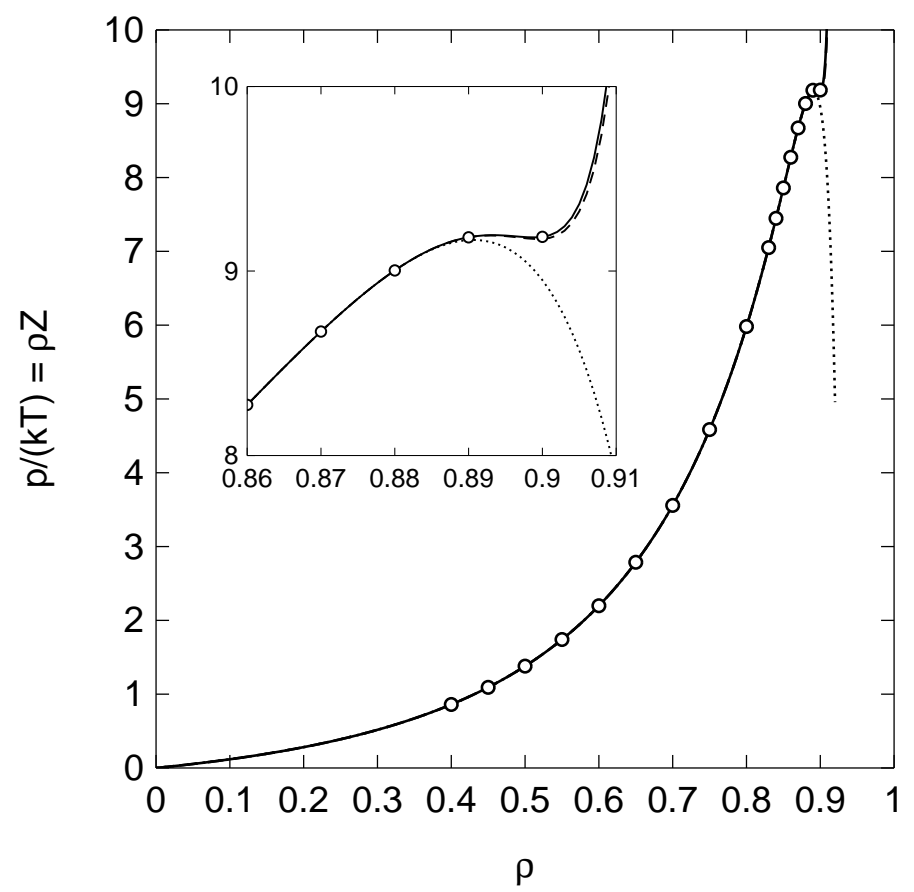

Figure 2. The data and proposed EOS. Dotted line: $\rho_{\max }=0.88$, dashed line: $\rho_{\max }=0.89$, solid line: $\rho_{\max }=0.90$.

large and not available with sufficient precision. Linear extrapolation of $Z(1 / N)$ was used instead; the final results thus cease precision. Point $\rho=0.88$ is a borderline between applicability of both approaches: The ensemble correction is large and its accuracy may affect the results, but the extrapolation gives less accurate data.

The final corrected MD data are collected in table 1 The data in the "difficult" region close to the phase transition agree well with recent extensive Monte Carlo data [4,3] with the exception of density $\rho=0.9$ closest to $\rho_{\mathrm{c}}$ where the $N=1024^{2}$ result $Z=10.212$ [3] is significantly larger than our $Z=10.206$; in this case one cannot assume linearity of the $Z(1 / N)$ dependence and our value is probably affected by higher-order finite size errors.

\subsection{Equations of state}

We present three best versions differing by the maximum density $\rho_{\max }$, number of fitted parameters and the value of the objective function $s$ (8), see figure 2. Note that $x=y /(1-y)$, where $y$ is the packing fraction.

$\rho_{\max }=0.88, s=0.724:$

$$
\begin{gathered}
Z=1+2 x+1.12801775 x^{2}+0.00181895291 x^{3}-0.0526134737 x^{4}+0.0504951668 x^{5}- \\
0.0325433846 x^{6}+0.0133946531 x^{7}+0.00174265604 x^{8}-0.00944632202 x^{9}+0.00851111768 x^{10}- \\
0.0035963525 x^{11}+0.000577345106 x^{12}-1.06399127 \cdot 10^{-7} x^{19}
\end{gathered}
$$

$\rho_{\max }=0.89, s=0.966$ :

$$
\begin{gathered}
Z=1+2 x+1.12801775 x^{2}+0.00181895291 x^{3}-0.0526134737 x^{4}+0.0504963915 x^{5}- \\
0.0325578581 x^{6}+0.0134816028 x^{7}+0.00129187484 x^{8}-0.00808881628 x^{9}+0.00669011963 x^{10}- \\
0.00250795961 x^{11}+0.000336036442 x^{12}-5.15282664 \cdot 10^{-9} x^{22}+5.57730095 \cdot 10^{-23} x^{57}
\end{gathered}
$$

$\rho_{\max }=0.90, \sigma=0.927$; region $\rho \in[0.89,0.90]$ of this equation may be affected by finite size effects:

$$
\begin{gathered}
Z=1+2 x+1.12801775 x^{2}+0.00181895291 x^{3}-0.0526134737 x^{4}+0.0504960168 x^{5}- \\
0.0325537792 x^{6}+0.0134578632 x^{7}+0.00140888182 x^{8}-0.00834273601 x^{9}+0.00694127367 x^{10}- \\
0.00262254723 x^{11}+0.000355746352 x^{12}-5.24672938 \cdot 10^{-9} x^{22}+5.88054639 \cdot 10^{-23} x^{57}
\end{gathered}
$$




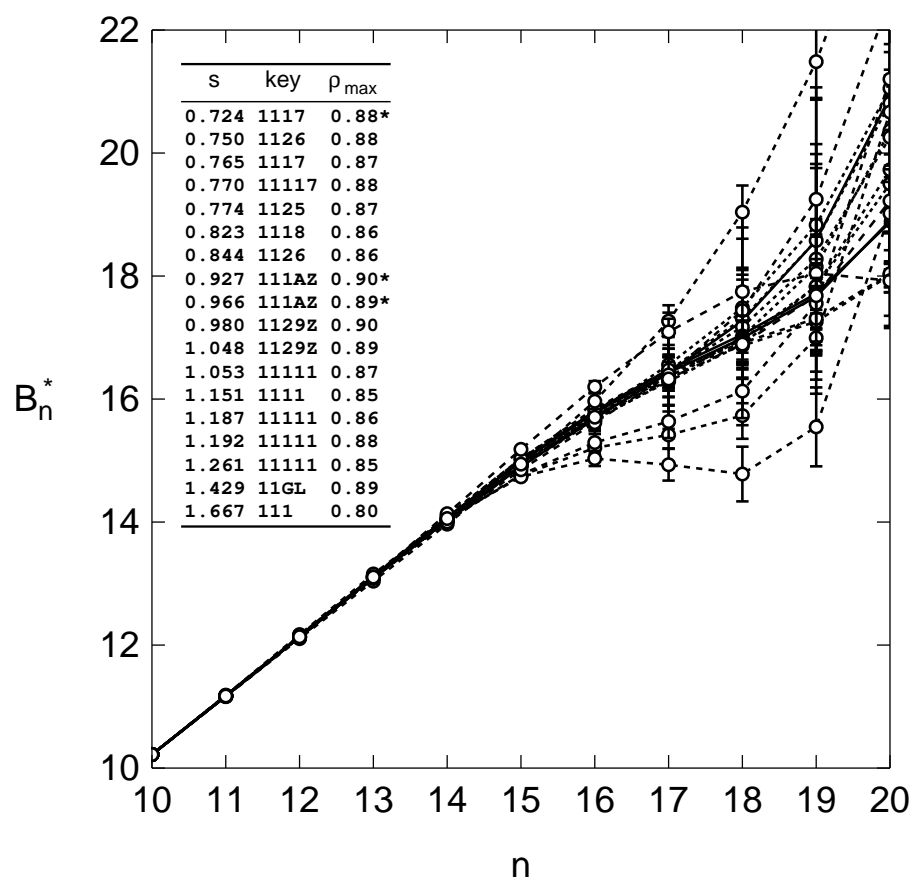

Figure 3. Higher-order virial coefficients as predicted by different EOSs. The best equations from Section 3.2 are marked by $\star$ in the table and by solid lines, otherwide the dash size is proportional to the value of $s$ (the longer dash, the worse fit). The key determines the equation: a digit $\left(\mathrm{A}=10\right.$, etc.) stands for one adjustable parameter (in addition to five parameters corresponding to $B_{6}$ to $B_{10}$ ), its value is the power of $y$ with respect to the previous term. Error bars are standard errors propagated from standard errors of the MD data and known virial coefficients.

It is interesting that both equations with $\rho_{\max } \geq 0.89$ predict to some extent the loop (with the "classical" critical exponent $\alpha^{\prime}=3$ ) at the critical (fluid/hexatic) point, even if this is not the aim of the present work which focuses rather on the low-density region. Any extrapolation to $\rho>\rho_{\mathrm{c}}$ should be done with caution because function $p(\rho)$ is likely nonanalytical at $\rho_{\mathrm{c}}$.

\subsection{Virial coefficients}

The virial coefficients were determined from a number of EOSs, see figure 3, The error of a predicted virial coefficient consists of a statistical error which is calculated from standard errors of input data and from a systematic (method) error. The former error ranges from typically 0.0014 (max. 0.002) for $B_{10}^{*}$ to typically 0.02-0.05 (max. 0.07) for $B_{14}^{*}$ to typically $0.04-0.2$ (max. 0.4) for $B_{16}^{*}$. The latter error is apparently difficult to determine. We believe that the range of different predictions, also those with $s>1$, gives a certain measure the systematic error; it also approximately matches the maximum statistical errors found in the used set od EOSs. We are rather pessimistic in determining this error; most EOSs with $s<1$ (thin lines in figure 3) give several times less scattering in the virials predicted and only moderately increased statistical errors. All virial coefficients are collected in table 2

To verify the procedure, we repeated the calculations with $B_{10}$ removed from the second sum of (8). The predicted value was $B_{10}=10.2210 \pm 0.002$, which is in agreement with the direct $\mathrm{MC}$ datum [5] (and in fact more accurate). The "best" value based on all available data (incl. $B_{10}$ of [5] ) is only slightly smaller, $B_{10}=10.2203 \pm 0.002$. In contrast, lower-order virials are accurate enough and including the MD data in the fit does not improve precision.

\section{Concluding remarks}

The proposed equations of state in the fluid region combine all available information - virial coefficients and simulation compressibility data. The equations may serve in perturbation theories. They are not meant as a replacement of physically-based (but less accurate) equations which are able to described more phases. 


\begin{tabular}{|c|c|c|}
\hline$n$ & $B_{n}^{*}$ & $\sigma\left(B_{n}^{*}\right)$ \\
\hline 2 & 2 & 0 \\
\hline 3 & 3.1280177516 & 0 (rounded) \\
\hline 4 & 4.2578544562 & 0 (rounded) \\
\hline 5 & 5.33689664 & 0.00000064 \\
\hline 6 & 6.3630259 & 0.0000109 \\
\hline 7 & 7.352077 & 0.000028 \\
\hline 8 & 8.318677 & 0.000061 \\
\hline 9 & 9.27234 & 0.00027 \\
\hline 10 & 10.2161 & 0.0041 \\
\hline $10^{\mathrm{a}}$ & 10.2203 & 0.002 \\
\hline $10^{\mathrm{b}}$ & 10.2210 & 0.002 \\
\hline 11 & 11.172 & 0.010 \\
\hline 12 & 12.132 & 0.03 \\
\hline 13 & 13.097 & 0.06 \\
\hline 14 & 14.053 & 0.08 \\
\hline 15 & 14.94 & 0.21 \\
\hline 16 & 15.7 & 0.4 \\
\hline \multicolumn{3}{|c|}{$\begin{array}{l}\text { a EOS-based prediction incl. } B_{10} \text { of }[5] \text {; } \\
\text { the recommended value. } \\
\text { b EOS-based prediction ( } B_{10} \text { of }[5] \text { not } \\
\text { used). }\end{array}$} \\
\hline
\end{tabular}

The equations enable prediction of higher-order virial coefficients with no additional assumption on their order-dependence. The results also witness about the "law of complexity conservation": The value of the tenth virial coefficient can be obtained with comparable precision both directly by diagrammatic techniques [5] and by simulations.

In order to obtain highly accurate MD data, it was necessary to take into account finite-size effects, which is especially peculiar close to the critical fluid/hexatic point. The "van der Waals-like" loop reported by several authors in this region can be semiquantitatively predicted by the concept of suppressed density fluctuations in the canonical ensemble.

\section{Acknowledgments}

This work was supported by the The Ministry of Education, Youth and Sports of the Czech Republic under the project LC512 (Center for Biomolecules and Complex Molecular Systems).

This paper was presented at The Seventh Liblice Conference on the Statistical Mechanics of Liquids (Lednice, Czech Republic, June 11-16, 2006). 


\section{References}

[1] J. J. Erpenbeck and W. W. Wood, J. Stat. Phys. 35, 321 (1984).

[2] J. J. Erpenbeck and W. W. Wood. In Statistical Mechanics, part B, ed. B. J. Berne (Plenum Press, 1977).

[3] C. H. Mak, Phys. Rev. E 73, 065104 (2006).

[4] A. Jaster, Phys. Rev. E 59, 2594 (1999).

[5] N. Clisby and B. M. McCoy, Pramana 64, 775 (2005); also E-print cond-mat/0410511

[6] S. Labík, J. Kolafa and A. Malijevský, Phys. Rev. E 71, 021105 (2005).

[7] H. Reiss, H. L. Frisch and A. Lebowitz, J. Chem. Phys. 31, 369 (1959).

[8] D. Henderson, Mol. Phys. 30, 971 (1975).

[9] J. L. Colot and M. Baus, Phys. Lett. A 119, 135 (1986).

[10] A. Santos, M. Lopez de Haro and S. B. Yuste, J. Chem. Phys. 103, 4622 (1995).

[11] F. C. Andrews, J. Chem. Phys. 62, 272 (1975).

[12] A. Baram, M. Luban, J. Phys. C 12, L659 (1979).

[13] L. V. Woodcock, J. Chem. Soc. Faraday Trans. 72, 731 (1976).

[14] J. J. Erpenbeck, M. Luban, Phys. Rev. A 32, 2920 (1985).

[15] I. C. Sanchez, J. Chem. Phys. 101, 7003 (1994).

[16] F. H. Stillinger, D. K. Stillinger, S. Torquato, T. M. Truskett and P. G. Debenedetti, J. Chem. Phys. 113, 10186 (2000).

[17] D. K. Stillinger, F. H. Stillinger, S. Torquato, T. M. Truskett and P. G. Debenedetti, J. Stat. Phys. 100, 49 (2000).

[18] E. Eisenberg and A. Baram, Phys. Rev. E 73, 025104 (2006).

[19] K. Binder, S. Sengupta and P. Nielaba, J. Phys. Cond. Mat. 14, 2323 (2002).

[20] A. Jaster, Europhys. Lett. 42, 277 (1998).

[21] J. Kolafa, S. Labík and A. Malijevský, Phys. Chem. Chem. Phys. 6, 2335 (2004).

[22] J. Kolafa, S. Labík and S. Malijevský, Molec. Phys., 100, 2629 (2002).

[23] L. R. Pratt and S. W. Haan, J. Chem. Phys. 741864 and 1873, (1981).

[24] J. L. Lebowitz and J. K. Percus, Phys. Rev. 124, 1673 (1961).

[25] F. L. Román, J. A. White and S. Velasco, J. Chem. Phys. 107, 4635 (1997).

[26] M. Metropolis, A. W. Rosenbluth, M. N. Rosenbluth, A. H. Teller, and E. Teller, J. Chem. Phys. 21, 1087 (1953).

[27] N. Clisby and B. M. McCoy, J. Stat. Phys. 114, 1343 (2004).

[28] K. W. Kratky, J. Stat. Phys. 29, 129 (1982).

[29] H. Reiss, H. L. Frisch and J. L. Lebowitz, J. Chem. Phys. 31, 369 (1959).

[30] E. Thiele, J. Chem. Phys., 39, 474 (1963).

[31] N. F. Carnahan and K. E. Starling, J. Chem. Phys. 51, 635 (1969).

[32] W. M. Gelbart and B. Barboy, J. Stat. Phys., 22, 709 (1980).

[33] I. C. Sánchez, J. Chem. Phys., 101, 7003 (1994).

[34] A. Malijevsky and J. Veverka, Phys. Chem. Chem. Phys. 1, 4267 (1999).

[35] J. Kolafa, Phys. Chem. Chem. Phys. 8, 464 (2006).

[36] K. Binder, Rep. Prog. Phys., 50, 783 (1987).

[37] S. N. Isaacs, Commun. Math. Phys., 95, 427 (1984); S. Friedli and C.-E. Pfister, Commun. Math. Phys., 245, 69 (2004). 